\title{
BMJ Open Improving the patient-centred care of children with life-altering skin conditions using feedback from electronic patient-reported outcome measures: protocol for a hybrid effectiveness-implementation study (PEDS-ePROM)
}

Zephanie Tyack (1) , , 2,3 Megan Simons, ${ }^{1,4}$ Steven M McPhail, ${ }^{3,5}$ Gillian Harvey, Tania Zappala, ${ }^{7}$ Robert S Ware (D) , ${ }^{8}$ Roy M Kimble ${ }^{1,2}$

To cite: Tyack Z, Simons M, McPhail SM, et al. Improving the patient-centred care of children with life-altering skin conditions using feedback from electronic patient-reported outcome measures: protocol for a hybrid effectivenessimplementation study (PEDS-ePROM). BMJ Open 2021;11:e041861. doi:10.1136/ bmjopen-2020-041861

- Prepublication history and supplemental material for this paper are available online. To view these files, please visit the journal online (http://dx.doi org/10.1136/bmjopen-2020041861).

Received 19 June 2020 Revised 24 December 2020 Accepted 23 February 2021

Check for updates

(c) Author(s) (or their employer(s)) 2021. Re-use permitted under CC BY-NC. No commercial re-use. See rights and permissions. Published by BMJ.

For numbered affiliations see end of article.

Correspondence to Dr Zephanie Tyack; z.tyack@qut.edu.au

\section{ABSTRACT}

Introduction Using patient-reported outcome measures (PROMs) with children have been described as 'giving a voice to the child'. Few studies have examined the routine use of these measures as potentially therapeutic interventions. This study aims to investigate: (1) the effectiveness of feedback using graphical displays of information from electronic PROMs (ePROMs) that target health-related quality of life, to improve health outcomes, referrals and treatment satisfaction and (2) the implementation of ePROMs and graphical displays by assessing acceptability, sustainability, cost, fidelity and context of the intervention and study processes. Methods and analysis A hybrid II effectivenessimplementation study will be conducted from February 2020 with children with life-altering skin conditions attending two outpatient clinics at a specialist paediatric children's hospital. A pragmatic randomised controlled trial and mixed methods process evaluation will be completed. Randomisation will occur at the child participant level. Children or parent proxies completing baseline ePROMs will be randomised to: (1) completion of ePROMs plus graphical displays of ePROM results to treating clinicians in consultations, versus (2) completion of ePROMs without graphical display of ePROM results. The primary outcome of the effectiveness trial will be overall health-related quality of life of children. Secondary outcomes will include other health-related quality of life outcomes (eg, child psychosocial and physical health, parent psychosocial health), referrals and treatment satisfaction. Trial data will be primarily analysed using linear mixed-effects models; and implementation data using inductive thematic analysis of interviews, meeting minutes, observational field notes and study communication mapped to the Consolidated Framework for Implementation Research.

Ethics and dissemination Ethical approval was obtained from Children's Health Queensland Human Research Ethics Committee (HREC/2019/QCHQ/56290), The University of Queensland (2019002233) and Queensland University
Strengths and limitations of this study

New evidence of the effectiveness and implementation of electronic patient-reported outcome measures in the routine clinical care of children with skin conditions and their parents will be generated which has received limited attention.

- Stakeholders representing multiple perspectives (children, parents, health professionals) were involved in the development of the intervention and process evaluation.

- Lack of ability to mask participants to the outcomes and contamination of the control group are potential biases, although child and parent participants were masked to the hypotheses.

of Technology (1900000847). Dissemination will occur through stakeholder groups, scientific meetings and peerreviewed publications.

Trial registration number Australian New Zealand Clinical Trials Registry (ACTRN12620000174987).

\section{INTRODUCTION}

The routine use of patient-reported outcome measures (PROMs), or proxy-report measures, as part of routine clinical care has been identified as a means of driving change in healthcare systems, to ensure the unique voice of the patient is heard. ${ }^{12}$ Potential benefits are improvements in shared decision-making, communication with health professionals and adherence to recommended treatments. ${ }^{3}$ PROMs are defined as questionnaires completed by a patient with a health condition about their own health and treatment. 
A recent systematic review identified that the effectiveness of PROM interventions for people with health conditions compared with usual care has been positive in adequately powered studies. ${ }^{4}$ Few trials have been conducted in children. Only 2 of 22 included randomised controlled trials were conducted in children, 1 focused on children with diabetes and 1 on children with cancer. ${ }^{56}$ Two more recent paediatric cluster randomised controlled trials investigated PROMs used with children with severe mental health conditions attending child and adolescent psychiatric services. ${ }^{78}$ Only one of the four paediatric trials identified positive effects of the PROM intervention. The positive effects were for psychosocial health-related quality of life but not physical healthrelated quality of life in children with diabetes. ${ }^{5}$

The implementation of PROMs in routine paediatric care has also recently been investigated in a systematic review, with increased identification and discussion around health-related quality of life reported, particularly in psychosocial and emotional domains, but with mixed results regarding the impact on the quality of care. ${ }^{9}$ Quality of care outcomes examined were satisfaction with treatment, referral rate and consultation length.

Implementation outcomes can be examined using an implementation science framework such as the Consolidated Framework for Implementation Research (CFIR). This framework has been identified as a 'good fit' for examining the implementation of PROMs in health service organisations in a recent systematic review of reviews that can assist to determine factors that influence implementation, ${ }^{10}$ and understand how the intervention works (ie, the process by which behaviour change occurs). ${ }^{11}$ Multi-level influences on implementation can be examined through a focus on individual characteristics of patients, families and clinicians (eg, knowledge and beliefs about the intervention), as well as organisational and process factors (eg, engagement). ${ }^{12}$

This paper will report the protocol for a randomised controlled trial and implementation study to test the effectiveness and implementation outcomes of a PROM feedback intervention targeting health-related quality of life, in children with the life-altering skin conditions of burn scars and infantile haemangiomas (termed the PEDS-ePROM study, where PEDS refers to children). The intervention involves the delivery of graphical displays of information from electronic PROMs (ePROMs) in routine consultations to encourage communication about the areas displayed and support clinical decision-making. A comparison intervention involves the completion of ePROMs alone without any graphical display of information. The need for interventions to improve the healthrelated quality of life of these children is highlighted by the lower health-related quality of life of children with burn scars across multiple domains even years after the actual injury compared with children with cancer. ${ }^{13}$ At the time of publication, the intervention had been designed and the randomised controlled trial and implementation testing was underway with no findings yet available.

\section{Aims and objectives}

The primary effectiveness aim is to determine the shortterm effectiveness of implementing ePROMs with graphical displays of result summaries, on overall health-related quality of life of children with life-altering skin conditions. Secondary aims will be to examine the effectiveness of the intervention for other health-related quality of life outcomes of children and parents, the number and type of referrals to health professionals and treatment satisfaction.

\section{Hypotheses (effectiveness component)}

1. The ePROM plus graphical display intervention will have a greater effect on overall health-related quality of life than the ePROM alone intervention, with a consistent direction and similar strength of effect across the clinics and conditions, supporting comparative effectiveness of the intervention.

2. The ePROM plus graphical display intervention will increase the number of psychosocial referrals to health professionals and increase parent proxy-reported satisfaction with treatment compared with the ePROM alone intervention.

\section{Implementation outcomes}

The primary aim is to determine the short-term acceptability and sustainability of implementing the interventions. The secondary aim is to determine the cost, fidelity and contextual factors related to implementation.

\section{METHODS AND ANALYSIS}

\section{Development of the study design and intervention}

The development of the PEDS-ePROM study and intervention was conducted from May 2019 to January 2020. We initiated preliminary discussion with clinicians in clinical areas to identify which measures were already being used routinely in practice. Systematic reviews and paediatric literature regarding the use of PROMs were also reviewed. Interview guides were developed to identify health outcomes that are meaningful and of high priority to children, their families and health professionals in the PROM intervention. ${ }^{14}$ The nine core questions from the International Society of Quality of Life user guide and the companion guide areas were addressed in the interviews. ${ }^{15}$ This development strategy using existing research and interviews with parent proxies and children has been identified as important to improve the engagement of children and young people such that fewer items are missed and responses accurately reflect their experiences and cognitive ability. ${ }^{16}$

Interviews were conducted with children with lifealtering skin conditions, their parents and treating health professionals in two phases as part of the preimplementation planning, with interview questions mapped to the CFIR. In the first phase the most appropriate outcomes and PROMs were identified. In the second phase the content validity of chosen PROMs and 
process evaluation outcomes were confirmed. Potential barriers and benefits to implementation were identified in both phases. For children with burn scars and their families, measures of health-related quality of life specific to scarring that included symptoms and treatment burden were prioritised based on conceptual work from the research team that identified these aspects as central components of health-related quality of life for this group. ${ }^{17}$ The design of the randomised controlled trial was based on systematic review findings that identified greater benefits when PROM results were provided to clinicians compared with when results were not provided to clinicians. ${ }^{4}$ Measures of the child's healthrelated quality of life were completed using parent-proxy and child self-report. The age cut-off for child self-report of 8 years or older was chosen for several reasons: this cut-off was being used in clinical practice in the burn scar clinics in the study setting; the burn scar-specific measures chosen were developed based on this cut-off; and the experience of the clinical and research team had identified that younger children aged 5-8 years often had difficulty comprehending the concepts captured in health-related quality of life measures. ${ }^{18}$ The difficulty children aged 5-8 years may have completing PROMs of health-related quality of life aligns with the findings of other paediatric researchers who identified the strongest evidence was for the broad age range of 6-8 years as the youngest age children can meaningfully report on a patient-reported outcome. ${ }^{19}$

\section{ePROM and graphical display intervention}

The Pediatric Quality of Life Inventory infant and generic scales $^{2021}$ measuring health-related quality of life were included as generic measures that were the same across the clinics and conditions. Condition-specific healthrelated quality of life measures were also included as these measures have been identified as being more responsive to change than generic measures. ${ }^{22}$ Condition-specific health-related quality of life measures selected were the Brisbane Burn Scar Impact Profile, ${ }^{17} 18$ The CARe parent scale ${ }^{23}$ Hemangioma Family Burden questionnaire ${ }^{24}$ and Infantile Hemangioma Quality of Life Scale. ${ }^{25}$ Selected measures targeted children and their parents and a single item targeted siblings. An open-ended option was also available for child and parent participants to report their priorities for care. Only PROMs meeting the criteria of content validity supported by involvement of the target group in development were included with the exception of the treatment satisfaction item. Graphical displays of result summaries from the Pediatric Quality of Life Inventory (PedsQL) and condition-specific measures of health-related quality of life measure will be presented in consultations for children with skin conditions and their parents to treating clinicians. The components of the intervention are reported in table 1.

\section{Method for completing PROMs}

Electronically delivered PROMs were identified as the best option for getting patients to complete the measures at home prior to consultations to reduce the burden of administration of measures and result summaries during busy clinics. The ePROMs will be administered via a weblink sent to parent participants in an email in the 3 days prior to their appointment. If the questionnaires are not completed via the weblink, child and parent participants will be offered a further opportunity to complete the questionnaires using an iPad prior to their consultation at the outpatient clinic while they are waiting for their consultation where possible. Parent proxy-report will continue throughout the study for any child who turns 8 years of age after first completion using parent proxy-report. Phone calls or text messages will be used to remind parent participants to complete the ePROMs. The ePROMs and graphical display of result summaries will be generated using the online survey software program Qualtrics ${ }^{26}$ and presented to treating health professionals immediately prior to appointments. Copies of the ePROMs and graphical displays of result summaries will be stored in medical records.

\section{Context}

The setting will be two outpatient clinics at a major metropolitan quaternary-level children's hospital in Australia; a burns clinic and a vascular anomalies clinic. Parents (and their children with skin conditions if aged 8 years or older) will be consecutively approached and recruited, and the intervention delivered prior to and at these clinics. The catchment of the hospital includes inhabitants from rural, regional and metropolitan areas including those from surrounding islands. Recruitment commenced in January 2020. The first participant was randomised to receive the intervention in March 2020.

\section{Research design}

A hybrid type 2 effectiveness-implementation design will be used which blends evaluating intervention effectiveness and understanding implementation of the intervention simultaneously. ${ }^{27}$ Benefits of this design include reduced lag time for uptake of the results into routine clinical practice and understanding the barriers and benefits to implementation. ${ }^{27} \mathrm{~A}$ pragmatic two-arm randomised controlled trial will be conducted using block randomisation in random blocks of 4, 6 or 8 stratified by diagnostic group (ie, infantile haemangiomas, burn scars), with child participants as the unit of randomisation; and an embedded qualitative process evaluation involving interviews with clinicians, and child and parent participants. The randomisation sequence will be prepared by a statistician independent from the study and will be concealed using sequentially numbered, opaque, sealed envelopes with tamper proof tape prepared by a person independent from the study.

The randomised controlled trial arms will be: (1) ePROM completion plus graphical display of result summaries to clinicians (intervention group) versus; (2) ePROM completion alone without graphical display of result summaries to clinicians (comparison group). 


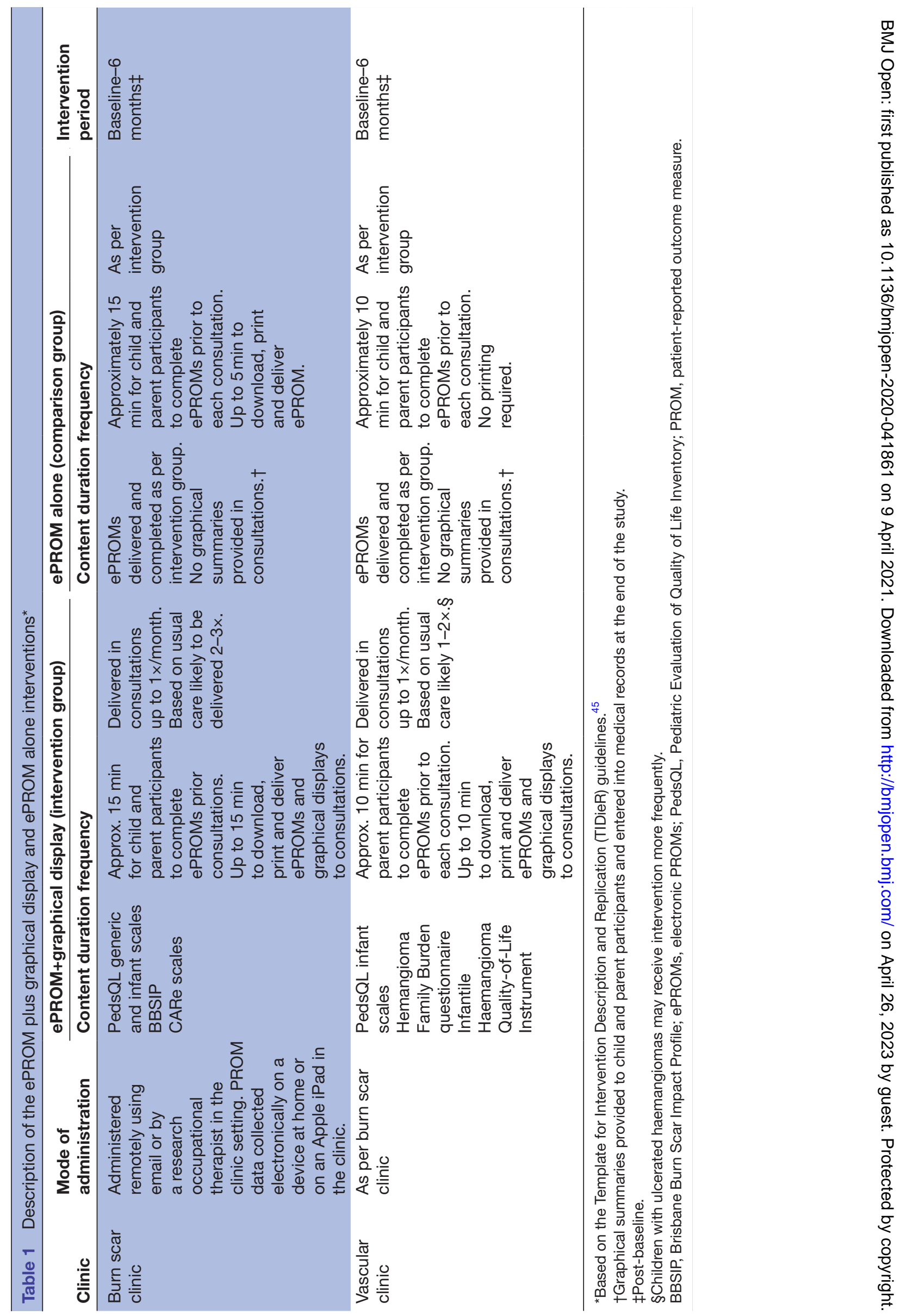




\begin{tabular}{|c|c|c|c|c|}
\hline \multicolumn{5}{|c|}{ STUDY PERIOD } \\
\hline \multirow[b]{2}{*}{ TIMEPOINT } & \multirow{2}{*}{$\begin{array}{c}\text { Enrolment } \\
t_{1}\end{array}$} & \multirow{2}{*}{$\begin{array}{c}\text { Allocation } \\
\mathbf{0} \\
\text { Baseline* }^{*} \\
\end{array}$} & \multicolumn{2}{|c|}{ Post-allocation } \\
\hline & & & $\begin{array}{c}t_{1} \\
\text { 3-months } \\
\text { post-baseline }\end{array}$ & $\begin{array}{c}t_{2} \\
\text { 6-months } \\
\text { post-baseline } \\
\end{array}$ \\
\hline \multicolumn{5}{|l|}{ ENROLMENT: } \\
\hline Eligibility screen & $x$ & & & \\
\hline \multirow{2}{*}{$\begin{array}{r}\text { Informed consent } \\
\text { Allocation }\end{array}$} & $x$ & $x$ & & \\
\hline & & $x$ & & \\
\hline \multirow{3}{*}{$\begin{array}{r}\text { INTERVENTIONS: } \\
\text { ePROM + graphical display of } \\
\text { results } \\
\text { ePROM only }\end{array}$} & & & & \\
\hline & & & & $\longrightarrow$ \\
\hline & & & & $\longrightarrow$ \\
\hline \multicolumn{5}{|l|}{ EVALUATIONS: } \\
\hline \multirow[t]{2}{*}{ Sociodemographic details } & & $x$ & & \\
\hline & & $x$ & & \\
\hline Clinical characteristics & & $x$ & $x$ & $x$ \\
\hline \multirow{2}{*}{$\begin{array}{l}\text { PedsQL (Infant \& generic scales) } \\
\text { CHU-9D (individual items \& utility } \\
\text { score) }\end{array}$} & & $x$ & $x$ & $x$ \\
\hline & & $x$ & $x$ & $x$ \\
\hline Brisbane Burn Scar Impact Profile** & & $x$ & $\mathrm{x}$ & $\mathrm{x}$ \\
\hline $\begin{array}{l}\text { Haemangioma Family Burden } \\
\text { Questionnaire }\end{array}$ & & $x$ & $x$ & $x$ \\
\hline \multirow{2}{*}{$\begin{array}{r}\text { Infantile Haemangioma Quality of } \\
\text { Life Instrument }\end{array}$} & & $x$ & $\mathrm{x}$ & $\mathrm{x}$ \\
\hline & & & $x$ & $x$ \\
\hline \multirow{2}{*}{$\begin{array}{r}\text { Referrals } \\
\text { Adverse effects }\end{array}$} & & & & $\longrightarrow$ \\
\hline & & $\longmapsto$ & & $\longrightarrow$ \\
\hline
\end{tabular}

Figure 1 Standard Protocol Items: Recommendations for Interventional Trials flow diagram for the effectiveness study component. *Baseline measures completed prior to randomization; $\geq 2$ nd appointment vascular clinic, $\geq 1$ st appointment scar clinic. PROMs, electronic, ${ }^{\star *}$ burn scar clinic only; ${ }^{* * *}$ vascular clinic only PROMs. CHU-9D, Child Health Utility - 9 item; Peds-QL, Pediatric Evaluation of Quality of Life Inventory; PROM, patient-reported outcome measure.

Baseline PROM measurement will occur before randomisation. PROM measurement will occur prior to or at one or more hospital appointments over the following 6 months and follow-up measurement will occur at 3 months and 6 months post-baseline if these timepoints differ from data collection timepoints during consultations with health professionals. Child and parent participants will be masked to the hypotheses. A Standard Protocol Items: Recommendations for Interventional Trials flow diagram has been used to report the schedule for enrolment, interventions and evaluations for the effectiveness component of the study (figure 1).

The study design and evaluation plan have been informed by the CFIR. This framework covers the physical and social environment, values, individual motivation and capacity factors which are considered important for the intervention being tested and has been derived from 33 theories relating to implementation. ${ }^{28}$ This protocol paper has been prepared following the eHealth Consolidated Standards of Reporting Trials guidelines. ${ }^{29}$

\section{Participants}

Participants for the effectiveness trial will be consecutively sampled. A previous study by the author team using this sampling in the study setting with the same population $^{3031}$ demonstrated representation of the burn scar study population. ${ }^{32}$ Participants for the implementation study component will be purposively sampled with representation of parents across both clinics, those who responded positively and negatively to the intervention, and children across different age-groups or their parents where possible.

\section{Inclusion criteria}

Children with burn scars and infantile haemangiomas, aged 0-16 years at the time of recruitment, who require ongoing management in the hospital setting, and their parents aged 18 years or older will be included. Ongoing management is defined as children who require one or more ongoing hospital consultations with clinicians at the study setting beyond baseline in the 6-month post-baseline intervention period for the prevention or management of skin conditions as determined by treating clinicians at baseline. Treating clinicians will also be asked to determine children's ability to complete PROMs electronically based on their physical condition and knowledge of the family (ie, to determine if bilateral hand burns would prevent sufficient movement of their hands to use an iPad).

\section{Exclusion criteria}

Children and parents will not be eligible to participate if they are involved with child protection services and it is difficult to obtain consent, where circumstances interfere with the participant's ability to give informed consent (ie, diminished understanding or comprehension), or where there is difficulty completing the PROMs due to difficulty speaking or understanding written English. Participants who have difficulty speaking or understanding written English will be excluded as it was difficult to anticipate in advance the languages that might be required for ePROMs due to the cultural diversity of patients seen in the setting; as multiple ePROMs were being administered (four with no or few translations available) with most not developed or tested using culturally diverse groups which is an important criteria for establishing cross-cultural validity, ${ }^{33}$ as funding was not available for purchasing available translations of up to US $\$ 500$ per translation; and as the interventions were not developed with these people thus it was unclear whether the interventions would meet the needs of these potential participants.

\section{Sample size estimate}

The sample size was based on recruitment feasibility. A retrospective audit of child and parent participants of clinic attendees suggested at least 35 participants in each clinic can be recruited in the intervention period. In terms of the effectiveness randomised controlled trial, if outcome data are available for 70 participants 
overall, then with $80 \%$ power we will be able to detect an effect size for the difference between-arms of $0.68 \mathrm{SD}$ units or greater for overall health-related quality of life at 6 months post-baseline (alpha $=0.05$ ). A between group difference of 0.68 is considered clinically meaningful at the individual level by expert clinicians, as a medium to large effect is regarded as offsetting the burden of completion of ePROMs to patients and families and supporting implementation routinely in clinics. To account for $20 \%$ attrition expected at 6-month follow-up based on a prior study with children and parents completing PROMs in the burns clinic setting, ${ }^{30}$ recruitment will continue until at least 88 participants have been randomised to groups. The sample size estimate was based on all participants with data available including parent proxy and child report data. A recent systematic review of health-related quality of life in children with burns identified that parent-proxy and child self-ratings were generally comparable based on generic and burn specific measures. ${ }^{35}$ This findings is supported by an additional two trials examining burn scar specific health-related quality of life in the burn scar clinic in this study which were not included in the systematic review. ${ }^{30}$ These trials identified similar health-related quality of life scores using proxy and child report and for children aged less than 8 years and older than 8 years.

Interviews will be conducted with the following groups during implementation with numbers of participants represented approximately equally for each clinic: children with a skin condition, their parents and treating health professionals. Interviews will continue until saturation (ie, the point at which no further dimensions, nuances or insights of issues are identified ${ }^{36}$ building on interview data generated pre-implementation. A greater number of child interviews will be required than parent and health professional interviews based on our previous experience of generally obtaining shorter interviews of 15-20 min in children with burn scars than with parents and health professionals.

\section{Evaluation}

Effectiveness outcomes

Study outcome measures will be self-completed by children aged 8 years or older and proxy-completed by parents for younger children. The primary outcome assessed will be change in the child's generic overall health across both clinics measured using the Pediatric Quality of Life Inventory (PedsQL 4.0 Generic Core and Infant Scales proxy-report total score).$^{2021}$ Secondary outcomes will be: (1) change in the child's psychosocial and physical health across both clinics measured using respective subscales of the Pediatric Quality of Life Inventory (PedsQL); (2) change in the child's generic health across both clinics measured using individual items of the Child Health Utility (CHU-9D) and utility score; ${ }^{37}$ (3) conditionspecific health-related quality of life of the child (overall impact, sensory intensity, sensory frequency, sensory impact, mobility, daily living, friendships and social interaction, appearance, emotional reactions, and physical symptoms) measured using respective subscales of the Brisbane Burn Scar Impact Profile (burn scar clinic group only); (4) condition-specific health-related quality of life of parents (worry and impact) measured using respective subscales of the Brisbane Burn Scar Impact Profile (burn scar clinic group only); (5) condition-specific health-related quality of life of the parent (psychosocial functioning, negative mood and self-worth) measured using the respective subscales of the CARe parent questionnaire (burn scar clinic group only); (6) conditionspecific health-related quality of life of the child (physical symptoms, social interactions, emotional functioning, psychosocial functioning) measured using respective subscales of the Infantile Hemangioma Quality of Life Scale (infantile haemangioma vascular clinic group only); (7) condition-specific health-related quality of life of parents (relationship and work, budget) measured using the relationships and work dimension and single budget item of the Hemangioma Family Burden questionnaire; (8) parent overall satisfaction with treatment; (9) number and type of referrals for the child and parent. Parent overall satisfaction with treatment was measured based on the finding that significantly more intervention patients reported satisfaction with overall care in a study of children with diabetes, which was the only paediatric study that examined this outcome in a recent systematic review. ${ }^{4}$ The number and type of referrals was included as an outcome based on the findings of three paediatric studies identified in a recent systematic review, in which two studies reported an increase in the referral rates in the intervention group, and one study identified no difference in referral rates between the intervention and control groups. ${ }^{9}$ A description of each of the outcomes and psychometric properties of outcomes are reported in online supplemental file 1. Adverse effects of the PROM interventions will be monitored using the self-report of parent and child participants (where appropriate), treating health professionals as well as by monitoring of the PROM data by investigators.

\section{Other outcomes}

Sociodemographic data collected from or about parents will include the parent's relationship to the child, level of education, ethnicity, work status, household income and postcode; and from children aged 8 years or older or parents about their children will include, gender, ethnicity, education level, scar location and comorbidities of the child participants. Clinical data collected from electronic medical records will be per cent total body surface area burned, percent full thickness burn, length of time post-burn, type of healing (eg, spontaneous skin healing vs split thickness graft), type of burn; and length of time to re-epithelialisation, medications and complications during the study period.

\section{Effectiveness evaluation}

Descriptive statistics will be used to report the characteristics of the sample. The number of participants excluded 
based on the exclusion criteria will also be reported (eg, difficulty speaking English). An intention to treat analysis will be the primary approach but per protocol analyses will be compared with the intention to treat approach to examine the effect of those who didn't receive the intervention as intended. The key sociodemographic and clinical characteristic data that will be examined for baseline differences between the groups will be age, gender, education, household income, socioeconomic status of the neighbourhood where the family reside based on postcode, severity of baseline symptoms and healthrelated quality of life, body location of the condition, visibility of the condition (scars on the head, neck, face or hands) and time since the skin condition commenced or injury occurred. Baseline differences in informant (parent proxy and child self-report) will also be examined between the groups.

\section{Effectiveness analysis}

Primary outcome comparison at 6-months post-baseline will be based on overall health from the Pediatric Evaluation of Quality of Life Inventory between the intervention and comparison group using linear mixed-effects models that account for repeated observations from the same child and clustering within clinics and within treating health professionals. Covariables will be included for potentially confounding variables if any differences between groups are identified for key sociodemographic, and clinical characteristics at baseline. The analysis population will consist of all participants who have analysable data. To investigate possible effects of informant, age and gender, a prespecified subgroup analyses of the primary and secondary health-related quality of life outcomes will be stratified by informant (proxy vs child report), child age (0-24 months vs 2-8 years vs $8+$ years; except for CHU-9D which will be $3-8$ years vs $8+$ years) and gender (male vs female) to determine whether effect differences exist based on these factors. A sensitivity analysis will be conducted to compare the results of the parent proxy versus child self-report where available.

A sensitivity analysis will also be conducted using imputation techniques to replace non-ignorable data that is considered to be missing at random over the follow-up period, to determine whether bias is likely in the complete case analysis. A further sensitivity analysis will investigate the possibility of imbalance in severity of health-related quality of life in the two clinics at baseline. As well as reporting the results for generic health-related quality of life across the clinics, we will also report after stratifying by clinic. Secondary outcome comparisons will be conducted at 6-months post-baseline using linear mixedeffects models where appropriate. Multi-level or nested hierarchical analysis will examine the effect of clinic and treating health professional effects by examining patient clustering within clinics, and surgeons and occupational therapists clustered within clinics. The amount and type of missing data will be reported using descriptive statistics. The maximum potential effect of the intervention with children will be analysed according to the treatment actually received (an 'as treated' analysis incorporating treatment dose received). Data analysis will be conducted using Stata V.16.0 (Statacorp).

\section{Implementation outcomes}

Implementation will be considered successful if graphical displays of result summaries are presented to treating clinicians immediately prior to more than $85 \%$ of consultations where a patient is randomised to receive a report, and if PROMs and summaries are filed in electronic medical records for more than $75 \%$ of patients eligible to have PROM data provided to treating clinicians in the intervention period. The implementation outcomes of acceptability and sustainability ${ }^{38}$ will be used to determine the overall success of the implementation. The implementation outcomes of acceptability, sustainability, cost, fidelity and contextual factors are detailed in table 2.

\section{Implementation evaluation}

Implementation outcomes will be evaluated using interviews; health service, administrative, clinical costings and missing data; observational field notes of meetings and each clinic attended or planned; meeting minutes and study emails; and fidelity reports. Acceptability is defined as the perception among stakeholders that a treatment, service, practice or innovation is agreeable or satisfactory. ${ }^{38}$ Sustainability is defined as the extent that a newly implemented treatment is maintained within a service setting's ongoing, stable operations. ${ }^{38}$ The ways in which the needs of people with difficulty speaking or understanding written English can best be addressed in the future will be explored in interviews as part of understanding acceptability and sustainability, as these groups were excluded from participation. The data from these sources will be mapped to the CFIR. ${ }^{28}$ This framework can be used to understand barriers and facilitators to implementing the intervention at the individual level, the organisational level and settings external to the organisation which can assist in determining the sustainability and potential scaling up of the intervention. ${ }^{28}$ Factors related to implementation delivery that might have impacted on the intervention effectiveness will also be examined to understand whether and how the expected outcomes were achieved, and the reasons for this.

Fidelity of the intervention will be taken from study records kept by researchers. Immediately after face-toface consultations parents and children (where appropriate) will be requested to verbally report the graphical display topics that were discussed during the consultation in the intervention group.

\section{Implementation analysis}

Interpretive description ${ }^{39}$ will be used to thematically analyse the data. This qualitative analysis uses elements from several other qualitative methodologies including phenomenology, grounded theory and ethnography without focusing on any specific technique. ${ }^{39}$ Interpretive 
Table 2 Description of the implementation outcomes

\begin{tabular}{|c|c|}
\hline Outcome & Detailed description of the outcome \\
\hline $\begin{array}{l}\text { Acceptability of the } \\
\text { interventions and evaluation* }\end{array}$ & $\begin{array}{l}\text { The acceptability of the ePROM interventions and } \\
\text { evaluation by families of children with health conditions } \\
\text { and treating clinicians including content, complexity, } \\
\text { delivery and relative advantage }{ }^{38} \text { and reflecting and } \\
\text { evaluating (including the ability to meet needs of people } \\
\text { who have difficulty speaking or understanding written } \\
\text { English in the future). }{ }^{*} \\
\text { 1. } \geq 80 \% \text { of families will take }<15 \text { min to complete the } \\
\text { ePROMs as previous research has identified that } \\
\text { PROMs that are fast to complete are most acceptable } \\
\text { to clinicians and families. }{ }^{46} \\
\text { 2. } \geq 50 \% \text { of families will complete ePROMs across all } \\
\text { scheduled consultations that were eligible to be } \\
\text { included in the study, where consultations eligible } \\
\text { to be included were limited to one consultation over } \\
\text { any } 1-\text { month period. Based on preintervention phase } \\
\text { interviews and field notes of what was considered } \\
\text { acceptable for ongoing implementation of the PROMs } \\
\text { routinely in clinical practice in the study clinics and } \\
\text { evidence indicating completion rates of } 75 \% \text { were } \\
\text { achieved for system-wide implementation of PROMs } \\
\text { at a Canadian children's hospital. }{ }^{47} \\
\text { 3. Phone or text reminders for PROM completion were } \\
\text { required in } \leq 50 \% \text { of families. This outcome was based } \\
\text { on feedback from clinicians in the pre-implementation } \\
\text { phase indicating that phone call reminders for this } \\
\text { type of intervention are a burden to clinicians and may } \\
\text { impact uptake by clinicians. } \\
\text { 4. Technology-related issues with graphical displays of } \\
\text { result summaries or ePROM completion were present } \\
\text { for } \leq 10 \% \text { of families across all eligible appointments. } \\
5 \text {. } \geq 75 \% \text { of participants eligible to have ePROM data } \\
\text { provided to treating clinicians had intervention } \\
\text { ePROMs and graphical displays filed in electronic } \\
\text { medical records. }\end{array}$ \\
\hline
\end{tabular}

Sustainability of ePROM interventions and evaluation modification of the intervention) was continued or planned to be continued in routine clinical practice at the end of the study, and barriers and facilitators of sustained use.

Cost

Fidelity

The cost of implementing the intervention for patients in the intervention and control groups based on resource use from the perspective of the health service. for skin treatment (eg, medicines, complementary treatments), and details of hospital presentations, will be included.

The extent to which the interventions were delivered and
The extent to which the ePROM intervention (or a

\section{Data type, source and analysis}

Quantitative: electronic study data and administrative data; descriptive analysis. Qualitative: interview and field note data; thematic analysis including mapping to CFIR innovation constructs (eg, relative advantage, adaptability, complexity, cost in the pre-implementation and implementation stages; and reflecting and evaluating, design quality and packaging, compatibility, and relative priority in the implementation phase).

Qualitative: interviews with child, parent and health professional participants and field notes; analysed using thematic analysis and mapping to CFIR (eg, knowledge and beliefs about the intervention, design quality and packaging, needs and resources).

Qualitative: interview data relating to cost. Quantitative: study and administrative data, medical records, hospital clinical costings Data for healthcare resource utilisation for cointerventions department data. received as intended.

1. Dose of the intervention: child and parent verbal report health professional participants and field notes. of the topics on the graphical displays of ePROM results that were discussed during the consultation in the intervention group, immediately after the consultation.

2. Dose of the intervention: percentage of eligible consultations for each participant where ePROM data was completed in advance of the consultation as scheduled.

3. The number (percentage) of participants randomised to receive graphical displays of result summaries vs the number of participants who actually had graphical displays of result summaries delivered to consultations.

4. Amount and type of missing intervention-related ePROM data on Qualtrics. ${ }^{26}$ 
Table 2 Continued

\begin{tabular}{lll}
\hline Outcome & Detailed description of the outcome & Data type, source and analysis \\
\hline Contextual factors & $\begin{array}{l}\text { Barriers and facilitators to multi-level implementation of } \\
\text { the intervention and the evaluation; at the individual level, } \\
\text { clinic level, hospital level and outside the hospital setting. }\end{array}$ & $\begin{array}{l}\text { Qualitative: interviews with child, parent and } \\
\text { health professional participants; and field notes } \\
\text { CFIR (eg, culture, networks and communication, } \\
\text { implementation cost). }\end{array}$ \\
\hline
\end{tabular}

${ }^{*}$ Children $\geq 8$ years will self-report; parents will provide proxy-reports for children aged $<8$ years except for satisfaction with treatment which will only be self-reported by parents.

CFIR, Consolidated Framework for Implementation Research; ePROMs, electronic PROMs; PROMs, patient-reported outcome measures.

description is ideal for applied clinical questions and analysis of a wide range of data sources..$^{39}$ The analysis builds on what is known in terms of current practices and structures of health services and what is known and not known. ${ }^{39}$ Data analysis will be conducted iteratively, concurrently with interviews, with analysis conducted during the implementation phase building on analysis of pre-implementation interviews. Framework analysis ${ }^{40}$ will then be applied deductively, mapping the qualitative and quantitative data (eg, verbatim quotes and descriptive statistics) to the predefined key constructs of the CFIR as overarching themes. The data will be organised into a framework matrix where columns are codes and rows are participants. ${ }^{40}$ This analysis is conducted across participants as well as within participants. Steps in framework analysis include familiarisation; indexing; charting and synthesising. ${ }^{40}$ Pre-implementation and post-implementation differences will be examined, and themes that emerge in addition to the CFIR constructs, will be added to the framework. Positive and negative participant quotes and descriptive data will be examined separately for each construct in the framework to determine influences on implementation and the strength of each construct, for each clinics as well as across clinics. ${ }^{41}$ Once mapping to the CFIR has been completed, data that applies to the implementation outcomes of acceptability, sustainability, fidelity and contextual factors will then be summarised.

Interviews will be audio recorded and transcribed verbatim by study personnel. Recordings will be stored in a coded form on a secure password protected folder within The University of Queensland until coding has been completed, accessible to two of the investigators and a research assistant. The credibility of the analysis will be checked using member checking of the interview data, independent coding of the data by two researchers of at least $20 \%$ of the data, triangulation of the results across participant groups (managers, treating health professionals, parent and child participants) and using field notes, and reflective journaling. Microsoft Excel (V.16, Microsoft Corporation) and NVivo (V.10, QSR International, Doncaster, Victoria, Australia ${ }^{42}$ ) will be used to organise and code the data.

\section{Electronic platform}

The electronic survey platform Qualtrics ${ }^{26}$ was chosen to administer the PROMs and to provide graphical displays of result summaries based on visual aesthetics of the graphical displays compared with other survey programmes and prior experience of the investigators using the programme. Features of the programme that were important for administration of the chosen surveys and study design were the ability to have open-ended text, email distribution, ability to send reminders, display longitudinal responses, a recoding values function, automated scoring functionality and links to NVivo software ${ }^{42}$ for coding open text responses.

\section{Patient and public involvement}

Children aged 8 years and older with life-altering skin conditions, parents of children with life-altering skin conditions and treating health professionals in the study setting were involved in all study phases including development of the intervention, process evaluation, study design and implementation evaluation. These stakeholder groups reported on the burden of the planned intervention, potential time required to participate and acceptability of follow-up intervals in pre-implementation interviews. Plans include forming a stakeholder reference group to inform the interpretation and sustainability of the study findings.

\section{DISCUSSION}

To our knowledge studies of PROM interventions have not previously focused on children with life-altering skin conditions. A pragmatic approach has been taken to maximise relevance to the clinical context including limiting exclusion criteria, and developing and delivering an intervention that has limited interference with the running of very busy outpatient clinics. If the intervention is shown to have promising short-term results then secondary prevention impacts particularly on the emotional health of parents may be likely and the benefits higher in the longer term which will be examined in the future.

An outcome of the proposed study may be refinement of the intervention based on mapping to the CFIR which may identify additional elements that should be 
considered. The findings will also likely inform the design of a multisite cluster effectiveness-implementation study of a patient-reported outcome measure intervention in these children which may reduce the risk of contamination bias. ${ }^{8}$ Information obtained will inform ongoing efforts in paediatric care to use PROMs as part of routine clinical care.

\section{Strengths and limitations}

Strengths of the study include the involvement of stakeholders representing multiple perspectives (children, parents, health professionals) in the development of the intervention and the process evaluation, and the focus of the intervention and process evaluation on health-related quality of life. The use of the CFIR is also a strength. Theory-based interventions tend to be more effective than non-theory based interventions. ${ }^{43}$

More specifically, the current study will seek to understand how the inner setting of the organisation (ie, organisational culture and structural characteristics) impacts on implementation which has been identified as a research gap. $^{10}$

The lack of masking of treating health professionals and participants in the randomised controlled trial is a limitation although masking is not possible as the outcomes are patient or proxy-reported and it will be clear to most participants when results are presented in consultations. However, child and parent participants will be masked to the hypotheses. Potential contamination bias has also been raised as a possibility in trials of this nature where several clinics within a facility are included, as treating health professionals' awareness of issues that should be focused on may be raised, diluting the impact of the intervention. ${ }^{44}$

A limitation is the lack of inclusion of children and parents who have difficulty speaking or understanding English. Further attention is required to develop and test ePROM interventions for families from specific cultural backgrounds which is a challenge in the study setting where people from diverse cultural backgrounds are seen. Specifically, people of Aboriginal and Torres Strait Islander descent were not involved in the development process thus the intervention and study design may not be acceptable for this group of people and should be established.

\section{ETHICS AND DISSEMINATION}

Ethical approval has been received from Children's Health Queensland Hospital and Health Service Human Research Ethics Committee (HREC/19/QCHQ/56290), The University of Queensland (2019002233) and Queensland University of Technology (1900000847). Written consent will be obtained from parent and treating health professional participants once written and verbal information has been provided. Parents will be encouraged to discuss the study with children who can communicate with their parents prior to consent being obtained.
Adverse effects will be reported to the Children's Health Queensland Hospital and Health Service and Human Research Ethics Committees.

\section{Author affiliations}

${ }^{1}$ Centre for Children's Burns and Trauma Research, Child Health Research Centre, The University of Queensland, Saint Lucia, Queensland, Australia

${ }^{2}$ Pegg Leditschke Children's Burns Centre, Children's Health Queensland, South Brisbane, Queensland, Australia

${ }^{3}$ Australian Centre for Health Services Innovation, Centre for Healthcare Transformation, School of Public Health and Social Work, Queensland University of Technology, Brisbane, Queensland, Australia

${ }^{4}$ Department of Occupational Therapy, Queensland Children's Hospital, South Brisbane, Queensland, Australia

${ }^{5}$ Clinical Informatics Directorate, Metro South Health, Brisbane, Queensland, Australia

${ }^{6}$ Adelaide Nursing School, The University of Adelaide, Adelaide, South Australia, Australia

${ }^{7}$ General Paediatrics and Dermatology Department, Queensland Children's Hospital, South Brisbane, Queensland, Australia

${ }^{8}$ Menzies Health Institute Queensland, Griffith University - GC Campus, Southport, Queensland, Australia

\section{Twitter Zephanie Tyack @tyack_z and Gillian Harvey @GillHar26}

Contributors ZT designed the study with input from SMM and RSW for the effectiveness evaluation, GH for the implementation evaluation and RMK and MS for integrating with existing clinical processes. ZT drafted the protocol and SMM, MS, TZ, RSW and RMK critically revised the manuscript.

Funding This work was supported by a Health Services Research grant from the Children's Hospital Foundation, Brisbane, grant number 50297. The funder had no input into the design or conduct of the study.

Competing interests ZT, MS and RMK developed the Brisbane Burn Scar Impact Profile which was included as a scar-specific measure in this study. MS and RMK were clinical staff members of the health service where the study will be conducted at the time of submission.

Patient consent for publication Not required.

Provenance and peer review Not commissioned; externally peer reviewed.

Supplemental material This content has been supplied by the author(s). It has not been vetted by BMJ Publishing Group Limited (BMJ) and may not have been peer-reviewed. Any opinions or recommendations discussed are solely those of the author(s) and are not endorsed by BMJ. BMJ disclaims all liability and responsibility arising from any reliance placed on the content. Where the content includes any translated material, BMJ does not warrant the accuracy and reliability of the translations (including but not limited to local regulations, clinical guidelines, terminology, drug names and drug dosages), and is not responsible for any error and/or omissions arising from translation and adaptation or otherwise.

Open access This is an open access article distributed in accordance with the Creative Commons Attribution Non Commercial (CC BY-NC 4.0) license, which permits others to distribute, remix, adapt, build upon this work non-commercially, and license their derivative works on different terms, provided the original work is properly cited, appropriate credit is given, any changes made indicated, and the use is non-commercial. See: http://creativecommons.org/licenses/by-nc/4.0/.

ORCID iDs

Zephanie Tyack http://orcid.org/0000-0003-3376-5731

Robert S Ware http://orcid.org/0000-0002-6129-6736

\section{REFERENCES}

1 De Rosis S, Cerasuolo D, Nuti S. Using patient-reported measures to drive change in healthcare: the experience of the digital, continuous and systematic PREMs observatory in Italy. BMC Health Serv Res 2020;20:315.

2 Basch E. Patient-Reported Outcomes - Harnessing Patients' Voices to Improve Clinical Care. N Engl J Med 2017;376:105-8.

3 Field J, Holmes MM, Newell D. PROMs data: can it be used to make decisions for individual patients? A narrative review. Patient Relat Outcome Meas 2019;10:233-41. 
4 Ishaque S, Karnon J, Chen G, et al. A systematic review of randomised controlled trials evaluating the use of patient-reported outcome measures (PROMs). Qual Life Res 2019;28:567-92.

5 de Wit M, Delemarre-van de Waal HA, Bokma JA, et al. Monitoring and discussing health-related quality of life in adolescents with type 1 diabetes improve psychosocial well-being: a randomized controlled trial. Diabetes Care 2008;31:1521-6.

6 Wolfe J, Orellana L, Cook EF, et al. Improving the care of children with advanced cancer by using an electronic patient-reported feedback intervention: results from the PediQUEST randomized controlled trial. J Clin Oncol 2014;32:1119-26.

7 Bickman L, Douglas SR, De Andrade ARV, et al. Implementing a measurement feedback system: a tale of two sites. Adm Policy Ment Health 2016;43:410-25.

8 de Jong RK, Snoek H, Staal WG, et al. The effect of patients' feedback on treatment outcome in a child and adolescent psychiatric sample: a randomized controlled trial. Eur Child Adolesc Psychiatry 2019;28:819-34.

9 Bele S, Chugh A, Mohamed B, et al. Patient-reported outcome measures in routine pediatric clinical care: a systematic review. Front Pediatr 2020;8:364.

10 Foster A, Croot L, Brazier J, et al. The facilitators and barriers to implementing patient reported outcome measures in organisations delivering health related services: a systematic review of reviews. $J$ Patient Rep Outcomes 2018;2:46.

11 Michie S, Carey RN, Johnston M, et al. From theory-inspired to theory-based interventions: a protocol for developing and testing a methodology for linking behaviour change techniques to theoretical mechanisms of action. Ann Behav Med 2018;52:501-12.

12 Ahmed S, Zidarov D, Eilayyan O, et al. Prospective application of implementation science theories and frameworks to inform use of PROMs in routine clinical care within an integrated pain network. Qual Life Res 2020. doi:10.1007/s11136-020-02600-8. [Epub ahead of print: 02 Sep 2020].

13 Maskell J, Newcombe P, Martin G, et al. Psychosocial functioning differences in pediatric burn survivors compared with healthy norms. J Burn Care Res 2013;34:465-76.

14 Spuls PI, Gerbens LAA, Simpson E, et al. Patient-Oriented Eczema Measure (POEM), a core instrument to measure symptoms in clinical trials: a Harmonising Outcome Measures for Eczema (home) statement. Br J Dermatol 2017;176:979-84.

15 Chan EKH, Edwards TC, Haywood K, et al. Implementing patientreported outcome measures in clinical practice: a companion guide to the ISOQOL user's guide. Qual Life Res 2019;28:621-7.

16 Kramer JM, Schwartz A. Reducing barriers to patient-reported outcome measures for people with cognitive impairments. Arch Phys Med Rehabil 2017;98:1705-15.

17 Simons M, Price N, Kimble R, et al. Patient experiences of burn scars in adults and children and development of a healthrelated quality of life conceptual model: a qualitative study. Burns 2016;42:620-32.

18 Tyack Z, Ziviani J, Kimble R, et al. Measuring the impact of burn scarring on health-related quality of life: development and preliminary content validation of the Brisbane burn scar impact profile (BBSIP) for children and adults. Burns 2015;41:1405-19.

19 Arbuckle R, Abetz-Webb L. "Not just little adults": qualitative methods to support the development of pediatric patient-reported outcomes. Patient 2013;6:143-59.

20 Varni JW, Limbers CA, Neighbors K, et al. The PedsQLTM infant scales: feasibility, internal consistency reliability, and validity in healthy and ill infants. Qual Life Res 2011;20:45-55.

21 Varni JW, Seid M, Knight TS, et al. The PedsQL 4.0 generic core scales: sensitivity, responsiveness, and impact on clinical decisionmaking. J Behav Med 2002;25:175-93.

22 Wiebe S, Guyatt G, Weaver B, et al. Comparative responsiveness of generic and specific quality-of-life instruments. J Clin Epidemiol 2003;56:52-60.

23 Griffiths C Harcourt D. Care Burn Scales - parent quality of life form version 12018

24 Boccara O, Méni C, Léauté-Labreze C, et al. Haemangioma family burden: creation of a specific questionnaire. Acta Derm Venereol 2015;95:78-82.

25 Chamlin SL, Mancini AJ, Lai J-S, et al. Development and validation of a quality-of-life instrument for infantile hemangiomas. J Invest Dermatol 2015;135:1533-9.
26 Qualtrics. Qualtrics: survey research suite, 2014. Available: http:// www.qualtrics.com/ [Accessed 16 Jun 2020]

27 Curran GM, Bauer M, Mittman B, et al. Effectiveness-implementation hybrid designs: combining elements of clinical effectiveness and implementation research to enhance public health impact. Med Care 2012;50:217-26.

28 Damschroder LJ, Aron DC, Keith RE, et al. Fostering implementation of health services research findings into practice: a consolidated framework for advancing implementation science. Implement $\mathrm{Sci}$ 2009;4:50.

29 Eysenbach G, CONSORT-EHEALTH Group. CONSORT-EHEALTH: improving and standardizing evaluation reports of web-based and mobile health interventions. J Med Internet Res 2011;13:e126.

30 Wiseman J, Ware RS, Simons M, et al. Effectiveness of topical silicone gel and pressure garment therapy for burn scar prevention and management in children: a randomized controlled trial. Clin Rehabil 2020;34:120-31.

31 Frear CC, Cuttle L, McPhail SM, et al. Randomized clinical trial of negative pressure wound therapy as an adjunctive treatment for small-area thermal burns in children. Br J Surg 2020;107:1741-50.

32 Stockton KA, Harvey J, Kimble RM. A prospective observational study investigating all children presenting to a specialty paediatric burns centre. Burns $2015 ; ; 41: 476-83.2015$.

33 Ravens-Sieberer U, Erhart M, Wille N, et al. Generic healthrelated quality-of-life assessment in children and adolescents: methodological considerations. Pharmacoeconomics 2006:24:1199-220.

34 Stevanovic D, Atilola O, Vostanis P, et al. Cross-cultural measurement invariance of adolescent self-report on the Pediatric Quality of Life Inventory ${ }^{\mathrm{TM}}$ 4.0. J Res Adolesc 2016;26:687-95.

35 Spronk I, Legemate CM, Polinder S, et al. Health-related quality of life in children after burn injuries: a systematic review. J Trauma Acute Care Surg 2018;85:1110-8.

36 Vasileiou K, Barnett J, Thorpe S, et al. Characterising and justifying sample size sufficiency in Interview-Based studies: systematic analysis of qualitative health research over a 15-year period. BMC Med Res Methodol 2018;18:148-48.

37 Stevens K. Valuation of the Child Health Utility 9D index. Pharmacoeconomics 2012;30:729-47.

38 Proctor E, Silmere H, Raghavan R, et al. Outcomes for implementation research: conceptual distinctions, measurement challenges, and research agenda. Adm Policy Ment Health 2011;38:65-76.

39 Thorne S. Interpretive description: qualitative research for applied practice. Second ed. New York: Routledge, 2016.

40 Gale NK, Heath G, Cameron E, et al. Using the framework method for the analysis of qualitative data in multi-disciplinary health research. BMC Med Res Methodol 2013;13:117-17.

41 Varsi C, Ekstedt M, Gammon D, et al. Using the consolidated framework for implementation research to identify barriers and facilitators for the implementation of an Internet-based patientprovider communication service in five settings: a qualitative study. $J$ Med Internet Res 2015;17:e262.

42 QSR International. NVivo 10 [software program]. Version 10. Available: http://www.qsrinternational.com/products_nvivo.aspx [Accessed 16 Jun 2020].

43 Albarracín D, Gillette JC, Earl AN, et al. A test of major assumptions about behavior change: a comprehensive look at the effects of passive and active HIV-prevention interventions since the beginning of the epidemic. Psychol Bull 2005;131:856-97.

44 Greenhalgh J, Dalkin S, Gooding K, et al. Functionality and feedback: a realist synthesis of the collation, interpretation and utilisation of patient-reported outcome measures data to improve patient care. Health Services and Delivery Research 2017:5:1-280.

45 Hoffmann TC, Glasziou PP, Boutron I, et al. Better reporting of interventions: template for intervention description and replication (TIDieR) checklist and guide. BMJ 2014;348:g1687.

46 Teela L, van Muilekom MM, Kooij LH, et al. Clinicians' perspective on the implemented KLIK PROM portal in clinical practice. Qual Life Res 2020;23.

47 Gerhardt WE, Mara CA, Kudel I, et al. Systemwide implementation of patient-reported outcomes in routine clinical care at a children's Hospital. Jt Comm J Qual Patient Saf 2018;44:441-53. 